REVISTA CIENCIAS BIOMÉ DICAS

PRESENTACIÓN DE CASOS CLÍNICOS

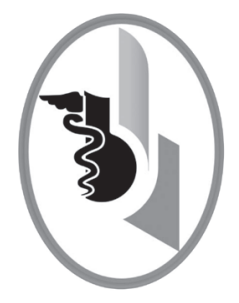

\title{
CARCINOMA UROTELIAL DE TRACTO URINARIO SUPERIOR: REPORTE DE UN CASO
}

UPPER TRACT UROTHELIAL CARCINOMA: CASE REPORT

\author{
Lambis-Ricardo Jorge ${ }^{1}$ \\ Herrera-Lomónaco Sandra² \\ Ballestas-Almario Carlos ${ }^{3}$ \\ Mendoza-Luna Alfredo ${ }^{3}$ \\ Montenegro-Castañeda Stephany ${ }^{4}$ \\ González-Peralta Aura ${ }^{4}$ \\ Correspondencia: aumagope@hotmail.com \\ Recibido para evaluación: marzo -20 - 2014. Aceptado para publicación: agosto - 15 - 2015.
}

\section{RESUMEN}

Introducción: los carcinomas uroteliales de tracto urinario superior primarios son tumores raros, representan el $5 \%$ de todos los cánceres uroteliales y menos del $10 \%$ de los tumores renales. Los síntomas son inespecíficos. Son más frecuentes en la 7-80 década de la vida. El pronóstico es pobre para los pacientes con enfermedad avanzada. Caso clínico: masculino de 55 años con hematuria microscópica y dolor lumbar. Ureterorrenoscopia flexible muestra sangrado difuso proveniente de las papilas de cáliz superior, asociado a lesión de características tumorales. Se realiza nefrectomía abierta izquierda con ureterotomía izquierda. Estudio anatomopatológico reportó carcinoma urotelial invasivo de tracto urinario superior. El paciente presentó evolución satisfactoria. Conclusión: el carcinoma urotelial del tracto superior es una enfermedad rara. Es importante la clasificación y estadificación preoperatoria precisa. El tratamiento de elección es la nefroureterectomía. Rev.cienc.biomed. 2015;6(2):364-368

\section{PALABRAS CLAVE}

Carcinoma urotelial; Pelvis renal; Nefroureterectomía radical; Ureteroscopia.

\section{SUMMARY}

Introduction: the primary upper urinary tract urothelial carcinomas are rare tumors, they represent $5 \%$ of all urothelial cancers and less than $10 \%$ of kidney tumors. The symptoms are nonspecific. They are more common in the seventh or eighth decade of life. The prognosis is poor for patients with advanced disease.

Case report: a 55 years old man with microscopic haematuria and lumbar pain. Supple ureterorenoscopy shows bleeding in napa that comes from papillae of upper calyx associated with tumor lesion characteristics. Open left nephrectomy with left urethrotomy was performed. Pathological study reported invasive upper urinary tract urothelial carcinoma. The patient presented satisfactory evolution.

\footnotetext{
Médico. Estudiante de Postgrado. Urología. Facultad de Medicina. Universidad de Cartagena. Colombia.

Médico. Especialista en Patología. Docente. Departamento de Diagnóstico. Facultad de Medicina. Universidad de Cartagena. Colombia.

Médico. Especialista en Urología. Docente. Departamento Quirúrgico. Sección Urología. Facultad de Medicina. Universidad de Cartagena. Colombia.

4 Estudiante de Medicina. Facultad de Medicina. Universidad de Cartagena. Colombia.
} 
ISSN: 2215-7840, 6(2), julio-diciembre 2015, Lambis-Ricardo Jorge, Herrera-Lomónaco Sandra, Ballestas-Almario Carlos, Mendoza-Luna Alfredo, Montenegro-Castañeda Stephany, González-Peralta Aura

Conclusion: the upper tract urothelial carcinoma is a rare disease. It is important classification and accurate preoperative staging. The treatment of choice is nephroureterectomy. Rev.cienc.biomed. 2015;6(2):364-368

\section{KEYWORDS}

Urothelial carcinoma; Kidney Pelvis; Radical nephroureterectomy; Ureteroscopy.

\section{INTRODUCCIÓN}

Los carcinomas uroteliales (CU) son la cuarta neoplasia maligna más común en los hombres después del cáncer de próstata, pulmón y colorrectal. Se pueden localizar en el tracto urinario inferior en relación con vejiga y uretra o en el tracto superior en relación con cavidades pielocaliciales y uréteres. Los tumores de localización vesical representan el 90-95\% de todos los tumores, siendo la ubicación más frecuente de esta neoplasia (1).

Por el contrario, los carcinomas uroteliales de tracto urinario superior (CUTUS) son tumores infrecuentes, con una incidencia estimada de 1 a 2 por cada 100.000 habitantes al año, lo cual representa solo el $5 \%$ de los CU y menos del $10 \%$ de los tumores renales $(1,2)$.

El grupo etario más afectado por los CUTUS es la séptima y octava década de la vida con una relación 3:1 hombre:mujer (3). La exposición a numerosos factores ambientales está asociada con el desarrollo de este tipo de cáncer (4), se incluyen el tabaquismo, la exposición ocupacional a aminas aromáticas y la irritación crónica secundaria a cálculos e infección $(1,4)$.

Generalmente los síntomas son escasos (5), entre ellos el más común es la hematuria (6), también suele presentarse dolor en flancos y masa lumbar $(7,8)$. El diagnóstico puede ser fortuito, sin embargo, casi siempre se necesita el concurso de varias herramientas diagnósticas $(1,9)$. La evaluación general comprende desde cistoscopia hasta ureteroscopía diagnóstica. El tratamiento de elección continúa siendo la nefroureterectomía radical con resección del rodete vesical. Recientemente algunos estudios han propuesto un tratamiento conservador (9). El objetivo fue presentar un caso clínico con manejo exitoso hasta un año de seguimiento de CUTUS.

\section{CASO CLÍNICO}

Se realizó seguimiento a paciente masculino de 55 años con enfermedad renal crónica, con deterioro moderado de la función renal que luego fue severamente disminuida. Según el riesgo relativo de la clasificación de la enfermedad renal crónica fue categorizada como G3b por lo cual no requería diálisis. El paciente presentó cuadro clínico de cuatro meses de evolución consistente en hematuria macroscópica anemizante y dolor lumbar.

Se realizó cistoscopia transuretral que evidenció eyaculación hematúrica por meato ureteral izquierdo. Se descartó hiperplasia prostática u otro hallazgo. Estudios adicionales como morfología eritrocitaria, UROTAC, ecografía renal y de vías urinarias presentaron parámetros normales. No fueron realizados estudios imagenológicos de contraste por estar contraindicados debido a su enfermedad renal crónica. Se realizó RMN que no reportó mayores hallazgos, el aortograma abdominal y arteriografía selectiva renal bilateral fue normal, razón por la que se descartó fístula arteriovenosa.

Además de ello, una ureterorrenoscopia flexible diagnóstica fue realizada con el fin de valorar trayecto ureteral donde se evidenció sangrado proveniente de las papilas de cáliz superior izquierdo, asociado a lesión de características tumorales en este nivel que luego se remitió a biopsia. Durante este procedimiento se realizó pielografía en la cual se observó pérdida de la arquitectura anatómica de los cálices superiores, lo que sugiere lesión maligna. Se decidió realizar nefrectomía abierta izquierda con ureterotomía izquierda. Estudio anatomopatológico señaló presencia de carcinoma urotelial invasivo del tracto urinario superior (Figura No 1 , No 2, No 3). El paciente presentó buena evolución y conservación de la función renal residual. Luego de un año de seguimiento continúa libre de recidivas. 


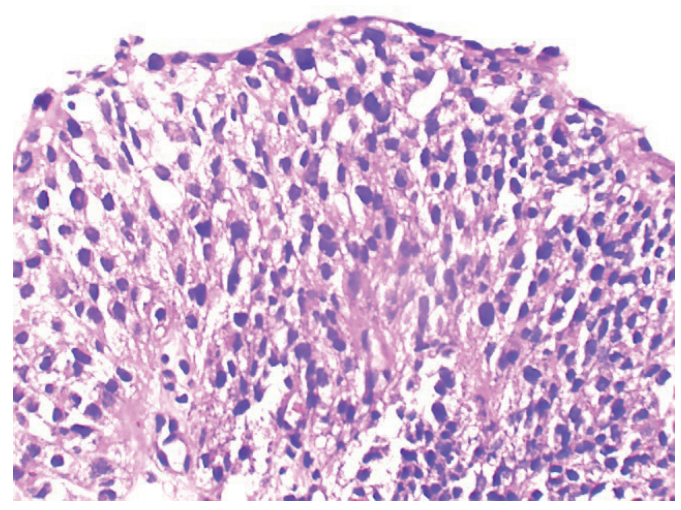

Figura No 1. H/E. Patrón sólido constituido por células transicionales de tamaño intermedio con núcleos hipercromáticos y escaso citoplasma.

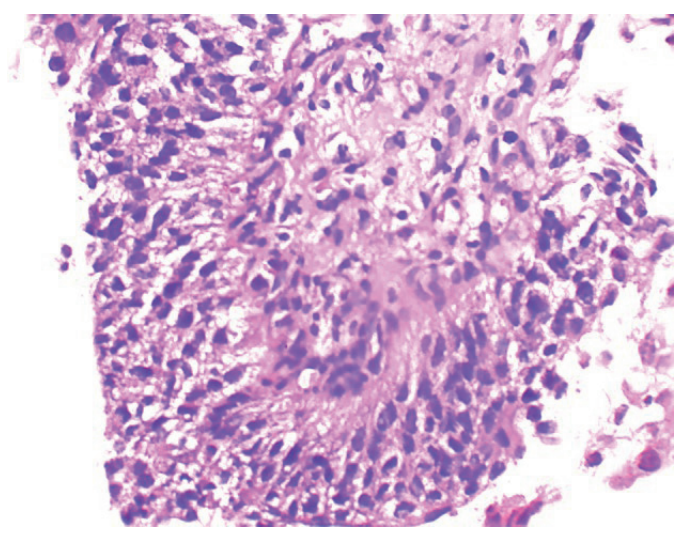

Figura No 2. H/E. Formaciones papilares tapizadas por células transicionales de tamaño intermedio.

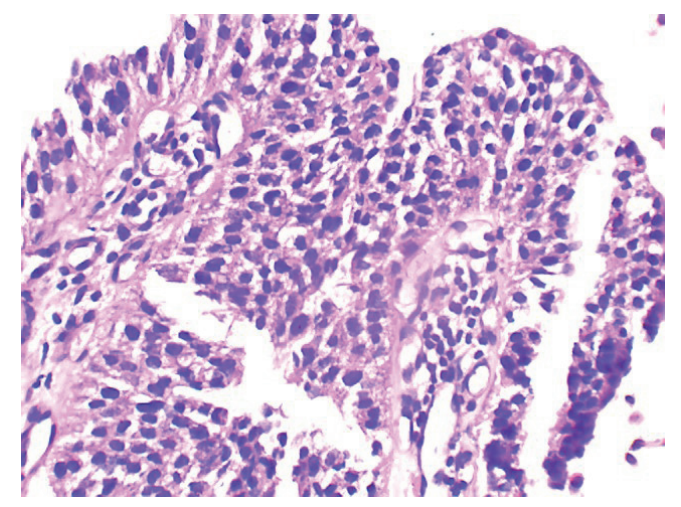

Figura No 3. H/E. Núcleos hipercromáticos y escaso citoplasma que compromete tres tercios del epitelio y que infiltran el parénquima renal.

\section{DISCUSIÓN}

Los CUTUS comprenden aproximadamente el $10 \%$ de las neoplasias renales y $5 \%$ de los carcinomas uroteliales (10). Esta patología tiene un pico de incidencia entre la séptima y octava década de la vida, donde los tumores pielocaliciales aproximadamente son dos veces más comunes que los tumores ureterales $(1,3)$. Muchos factores de riesgo ambientales se han asociado con el desarrollo de esta patología, entre los que se destaca el tabaco y la exposición ocupacional a aminas aromáticas tales como Benzidina y B- hNaftaleno $(4,11)$. También se ha descrito una asociación con la Nefropatía Endémica de los Balcanes, una enfermedad renal caracterizada por falla renal crónica y fibrosis tubulointersticial $(10,12)$.

Los síntomas de CUTUS son generalmente pocos, el más común es la hematuria macroscópica o microscópica (70-80\%), el dolor en flanco se presenta en un $20-40 \%$ de los casos y una masa renal está presente en el $10-20 \%$ de los mismos (1).

Macroscópicamente el carcinoma urotelial de tracto superior puede ser solitario o multifocal, puede observarse un crecimiento papilar o sésil, masas sólidas, nodulares, ulceradas o difusamente infiltrantes. Es frecuente observar áreas de carcinoma urotelial no invasivo. La evidencia macroscópica de invasión puede ser detectada por el engrosamiento del uréter y/o de la pelvis renal, así como por la formación de un tumor solido en el parénquima renal (13). Algunos de estos tumores pueden ser grandes y alcanzar un tamaño de $9 \mathrm{~cm}$, sin embargo, el tamaño promedio es de $3.7 \mathrm{~cm}$ (14). En nuestro caso el tumor se encontraba dentro del tamaño promedio, exhibiendo crecimiento exofítico en las papilas del cáliz superior.

Microscópicamente las variantes morfológicas descritas de los CUTUS en su mayoría están constituidas por células transicionales y corresponden siempre a tumores de alto grado, a diferencia de los carcinomas de vejiga que pueden ser de bajo grado $(14,15)$. Las variantes más frecuentemente asociadas a los CUTUS son: micropapilar de células claras, neuroendocrino y linfoepitelial (15). En nuestro caso el carcinoma urotelial fue de alto grado, con invasión a parénquima renal, cápsula renal y tejido perirrenal.

Un porcentaje importante de pacientes (45\%) con CUTUS tienen enfermedad localmente avanzada (mayor o igual que pT2) al 
ISSN: 2215-7840, 6(2), julio-diciembre 2015, Lambis-Ricardo Jorge, Herrera-Lomónaco Sandra, Ballestas-Almario Carlos, Mendoza-Luna Alfredo, Montenegro-Castañeda Stephany, González-Peralta Aura

momento de la nefrectomía (14). Aproximadamente el $2 \%$ de los CUTUS son bilaterales y cerca del $50 \%$ puede desarrollar posteriormente carcinoma urotelial de vejiga. Por el contrario, la incidencia de los CUTUS es baja (0.7-4\%) dentro de los pacientes con cáncer de vejiga primario. Se ha reportado un riesgo incrementado en pacientes con una historia familiar de cáncer colónico no polipósico y hereditario (16).

El factor pronóstico más importante para los pacientes CUTUS es la estadificación TNM $(14,17)$. Las características patológicas adicionales que pueden ser predictores independientes de la evolución clínica después de la nefroureterectomía radical son necrosis tumoral extensa, arquitectura del tumor sésil e invasión linfovascular (17). El paciente presentado tenía una lesión sólida de alto grado en contacto con la cápsula, sin embargo ha tenido buena evolución clínica y tras un año de seguimiento está libre de recidivas.

Generalmente la detección de los CUTUS no puede lograrse por una única herramienta diagnóstica (9). La evaluación estándar incluye cistoscopia, estudios de imagen del tracto urinario superior y la citología de orina, seguido de pielografía retrógrada, endoscopia y biopsia (18). El régimen con cistoscopia, citología urinaria, imagen del tracto superior y pielografía retrógrada puede establecer el diagnóstico en el 50 a $60 \%$ de los pacientes.
La precisión aumenta a $80-90 \%$ con la ureterorrenoscopia (19).

El tratamiento estándar para los CUTUS es la nefroureterectomía radical con resección del rodete vesical. Recientemente, algunos estudios proponen el tratamiento conservador con la ablación endoscópica láser como un tratamiento seguro y eficaz en casos seleccionados con enfermedad de bajo grado y estadio clínico bajo (20). La clasificación y estadificación precisa preoperatoria es de gran importancia debido a la multifocalidad, y capacidad de recurrencia. Suelen ser más agresivos que el carcinoma urotelial de vejiga (9).

\section{CONCLUSIÓN}

Los CUTUS pueden ser de difícil diagnóstico. Se recomienda el uso de la pielografía retrógrada asociada a ureterorrenoscopia principalmente porque permite realizar biopsias inmediatamente. El tratamiento preferido continúa siendo la nefroureterectomía radical.

CONFLICTO DE INTERESES: ninguno que declarar.

FINANCIACIÓN: recursos propios de los autores. Estudios de laboratorio, insumos hospitalarios, medicamentos y honorarios profesionales, aportados dentro de la atención asistencial.

\section{REFERENCIAS BIBLIOGRÁFICAS}

1. Roupret M, Babjuk M, Comperat E, Zigeuner R, Sylvester R, Burger M, et al. European guidelines on upper tract urothelial carcinomas: 2013 update. European urology. 2013;63(6):105971. Epub 2013/04/02.

2. Patel N, Arya M, Muneer A, Powles T, Sullivan M, Hines J, et al. Molecular aspects of upper tract urothelial carcinoma. Urologic oncology. 2014;32(1):28 e11-20. Epub 2013/02/23.

3. Shariat SF, Favaretto RL, Gupta A, Fritsche HM, Matsumoto K, Kassouf W, et al. Gender differences in radical nephroureterectomy for upper tract urothelial carcinoma. World journal of urology. 2011;29(4):481-6. Epub 2010/10/05.

4. Colin P, Koenig P, Ouzzane A, Berthon N, Villers A, Biserte J, et al. Environmental factors involved in carcinogenesis of urothelial cell carcinomas of the upper urinary tract. BJU international. 2009;104(10):1436-40. Epub 2009/08/20.

5. Inman BA, Tran VT, Fradet Y, Lacombe L. Carcinoma of the upper urinary tract: predictors of survival and competing causes of mortality. Cancer. 2009;115(13):2853-62. Epub 2009/05/13.

6. Cowan NC. CT urography for hematuria. Nature reviews Urology. 2012;9(4):218-26. Epub 2012/03/14.

7. Ito Y, Kikuchi E, Tanaka N, Miyajima A, Mikami S, Jinzaki M, et al. Preoperative hydronephrosis grade independently predicts worse pathological outcomes in patients undergoing nephroureterectomy for upper tract urothelial carcinoma. The Journal of urology. 2011;185(5):1621-6. Epub 2011/03/23. 
8. Raman JD, Shariat SF, Karakiewicz PI, Lotan Y, Sagalowsky AI, Roscigno M, et al. Does preoperative symptom classification impact prognosis in patients with clinically localized upper-tract urothelial carcinoma managed by radical nephroureterectomy? Urologic oncology. 2011;29(6):716-23. Epub 2010/01/09.

9. Tai Y-S, Chiang IN, Huang C-Y, Tai H-C, Pu Y-S. Effectiveness of different diagnostic tools for upper urinary tract urothelial carcinoma. Urological Science. 2015;26(1):57-60.

10. Remzi M, Shariat S, Huebner W, Fajkovic H, Seitz C. Upper urinary tract urothelial carcinoma: what have we learned in the last 4 years? Therapeutic Advances in Urology. 2011;3(2):69-80.

11. McLaughlin JK, Silverman DT, Hsing AW, Ross RK, Schoenberg JB, Yu MC, et al. Cigarette smoking and cancers of the renal pelvis and ureter. Cancer research. 1992;52(2):254-7. Epub 1992/01/25.

12. Grollman AP, Shibutani S, Moriya M, Miller F, Wu L, Moll U, et al. Aristolochic acid and the etiology of endemic (Balkan) nephropathy. Proceedings of the National Academy of Sciences of the United States of America. 2007;104(29):12129-34. Epub 2007/07/11.

13. Humphrey PA. Urothelial carcinoma of the upper urinary tract. The Journal of urology. 2014;192(4):1223-4. Epub 2014/07/22.

14. Olgac S, Mazumdar M, Dalbagni G, Reuter VE. Urothelial carcinoma of the renal pelvis: a clinicopathologic study of 130 cases. The American journal of surgical pathology. $2004 ; 28(12): 1545-$ 52. Epub 2004/12/04.

15. Perez-Montiel D, Wakely PE, Hes O, Michal M, Suster S. High-grade urothelial carcinoma of the renal pelvis: clinicopathologic study of 108 cases with emphasis on unusual morphologic variants. Modern pathology : an official journal of the United States and Canadian Academy of Pathology, Inc. 2006;19(4):494-503. Epub 2006/02/14.

16. Bostwick DG, Cheng L. Urologic Surgical Pathology. 2 ed: Elsevier Health Sciences; 2008.

17. Chromecki TF, Bensalah K, Remzi M, Verhoest G, Cha EK, Scherr DS, et al. Prognostic factors for upper urinary tract urothelial carcinoma. Nature reviews Urology. 2011;8(8):440-7. Epub 2011/07/06.

18. Painter DJ, Timoney AG, Denton K, Alken P, Keeley FX, Jr. The modern management of upper urinary tract urothelial cancer: tumour diagnosis, grading and staging. BJU international. 2007;99(5):973-7. Epub 2007/04/18.

19. Streem SB, Pontes JE, Novick AC, Montie JE. Ureteropyeloscopy in the evaluation of upper tract filling defects. The Journal of urology. 1986;136(2):383-5. Epub 1986/08/01.

20. Painter DJ, Denton K, Timoney AG, Keeley FX. Ureteroscopic management of upper-tract urothelial cancer: an exciting nephron-sparing option or an unacceptable risk? Journal of endourology / Endourological Society. 2008;22(6):1237-9. Epub 2008/06/27.

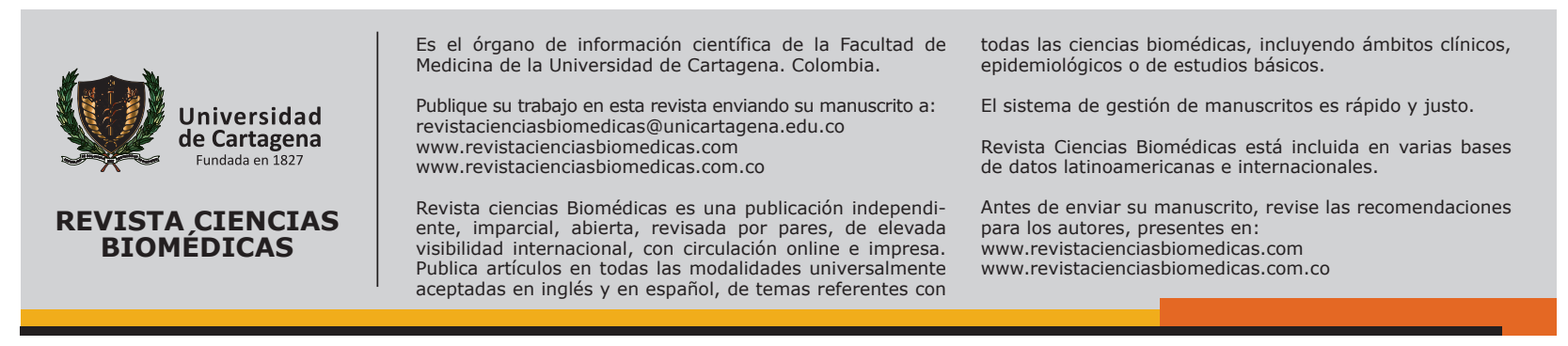

